solidus has been found. If the solid solutions show positive deviations from ideality, it would be expected that at low temperatures the two substances would be insoluble. A calculation (reported in an account ${ }^{2}$ of a meeting of the Physical Society Low Temperature Group). assuming that the intermolecular force of attraction is of the van der Waals type, shows that the separation into two phases would not occur in the temperature-range used in this investigation.

The experimental details and the results of the vapour pressure measurements will be discussed in a later publication.

I thank Prof. G. O. Jones, who suggested this investigation, for his continued help and advice.

\section{R. Heastie}

Department of Physics,

Queen Mary College,

London, E.I. June 6.

1 Veith, H., and Schröder, E., Z. phys. Chem., A, 179, 16 (1937). 2 Domb, C., Nature, 175, 661 (1955).

\section{Serially Balanced Sequences}

WILLIAMS ${ }^{1}$ has devised experimental designs for use when several subjects aro to be tested on successive occasions, and the response to the treatments on any one occasion may be modified by residual influences of the one or two previous treatments. We have recently considered the possibility of analogous designs that, under certain assumptions about the mathematical model, could be used for an experimental comparison of treatments on many occasions with one subject only. The practical importance of these designs is probably not great, but they are of interest as introducing new combinatorial problems.

Suppose that four treatments are represented by the letters $A, B, C, D$. Consider the sequence

$$
A ; B, C, A, D ; A, C, B, D ; C, D, B, A .
$$

This is an example of a serially balanced sequence for four treatments. More generally, for $t$ treatments, a serially balanced sequence is defined as follows: (i) each treatment follows each other treatment once (hence the whole sequence has $\left(t^{2}-t+1\right)$ members, one letter being repeated $t$ times and the others $(t-1)$ times each); (ii) after the first treatment, each block of $t$ successive treatments contains one complete replicate. The first and last treatments must be the same. From any sequence, $(t-2)$ new ones can be generated by cyclic permutation of whole blocks of four and alteration of the first letter to the new last letter. From each of these, a further $(t-1)$ can be generated by discarding the first letter, reversing the order of the remaining $t(t-1)$, and inserting a new first lotter. For $t=4$, sequences can be classified into three transformation sets, all members of a set being related by block permutation, reversal and letter permutation.

Statistical analysis according to the standard additive model is practicable if the time trend can be adequately represented by $(t-2)$ parameters for differences between the $(t-1)$ blocks; the first treatment is regarded purely as a conditioning of the subject, and no use is made of observations on it. The immediate effects of treatments and their residuals at the time of the next treatment each require $(t-1)$ parameters, leaving $(t-1)(t-3)$ degrees of freedom for the estimation of the error variance. If a longer experiment is required, so as to increase replication and to improve the estimate of error, two or more sequences can be used consecutively provided that all begin with the same letter: for each one after the first, this initial letter is telescoped with the last letter of the previous sequence.

Despite the balance, non-orthogonality makes the analysis awkward. For some values of $t$ (but not for $t=4$ ), additional balance can be achieved by having each block also contain one residual from each treatment. For example, with $t=5$, the sequence

$$
\begin{gathered}
A ; B, D, E, C, A ; E, B, C, D, A ; \\
C, E, D, B, A ; D, C, B, E, A
\end{gathered}
$$

is such that both the first $t(t-1)$ and the last $t(t-1)$ letters form $(t-1)$ successive replicates.

For most experimental purposes, a more complete form of serial balance, in which every letter follows every letter including itself once, and which therefore requires a sequence of $\left(t^{2}+1\right)$ letters, is obviously preferable. However, such sequences appear to be much rarer. None exists for $t=3,4,5$; for $t=6$ there are plenty, one such being

$$
\begin{aligned}
A ; & A, B, C, D, E, F ; F, C, E, A, D, B ; \\
& B, A, F, E, D, C ; C, A, E, B, F, D ; \\
& D, F, A, C, B, E ; E, C, F, B, D, A .
\end{aligned}
$$

Cyclic permutation of blocks and reversal again generate new sequences.

Further study of these sequences is in progress, and a paper describing their application in bioassay is in preparation.

This communication was prepared without know. ledge of a paper by R. M. Williams ${ }^{2}$ in which analogous designs were discussed; Williams, however, used a less severe constraint by balancing treatments in respect of adjacent treatments on either side instead of the preceding treatment only.

\section{J. FINNEY}

Anne D. OUthwarte

Department of Statistics,

University of Aberdeen. June 6.

${ }^{1}$ Williams, E. J., Australian Journal of Scientific Research, A, 2, 149 (1949) A. J., A Astralian Jour

"Williams, R. M., Biometrika, 39, 151 (1952).

\section{Corrosion and Meteorology}

In studies of corrosion under tropical conditions, it has for a long time been the practice to quote average yearly conditions of temperature and humidity. Although laboratory or simulated tropical testing is often carried out under cyclic conditions, these cannot be truly representative of the climatic conditions unless a complete examination of the climatic conditions is first made. This is particularly important in view of the principle of critical humidity developed by Vernon, Hudson, Patterson and Hebbs, and others ${ }^{1}$, and also the work of Clarke ${ }^{2}$ on fluctuating humidity.

An analysis of the climatic conditions in Singapore during $1953^{3}$ gave the following information on the percentage relative humidity per day during each month : 